\title{
Germination of radish seeds (Raphanus sativus) treated with homeopathic drugs.
}

\author{
Marcelo Felipe Bezerra Donadon¹, Euclides Davidson Bueno Romanoํ, \\ Walkiria Ruiz de Pinho ${ }^{1}$, Marina Lopes Vieira de Souza ${ }^{1}$, Pedro Henrique \\ Alcalde do Nascimento ${ }^{1}$, Maria do Rocio Lázaro Rodrigues ${ }^{2}$, Solange Monteiro \\ de Toledo Piza Gomes Carneiro ${ }^{1}$
}

(1) Agricultural Research Institute of Paraná, Londrina, PR, Brazil

(2) Center of Specialization on Homeopathy, Londrina, PR, Brazil

\begin{abstract}
The radish is a short cycle crop, since it is harvested at 25-30 days after direct sowing. Under the economic point of view it is an important species, but there are few studies on germination of radish seeds. The objective of this study was to evaluate the effect of high diluted substances on the germination of radish seeds. The trial was conducted at the Agricultural Research Institute of Paraná (IAPAR) in Londrina / Paraná. It was used a pesticide free cultivar named Cometa. The treatments were: Bryonia, hydroalcoholic solution, Arnica montana, Cina and Lupine + Oat, all of them diluted and agitated at 9x; distilled water and agitated distilled water were used as controls. The mother tincture of Lupine + Oat was prepared from plants collected at the experimental station of IAPAR in Londrina. The mother tincture and all treatments were prepared according to the guidelines in the Brazilian Homeopathic Pharmacopeia, Part I [1]. The agitations of the treatments were made by a mechanical equipment, model Denise 10-50 manufactured by Autic. The water was distilled the day before preparing the treatments. The experiment was performed with 300 seeds per treatment. The seeds were placed in gerbox with germitest paper, and 50 gerbox were used per treatment, with six seeds in each gerbox. The germitest papers were moistened with the treatments and the seeds were soaked for 2 hours previously set up the experiment. The substances in high dilutions were agitated 100 times on the machine before soaking the seeds and moistening the germitest paper. The experimental design was entirely randomized and the gerbox were kept at the bench at the Laboratory of Plant Protection of IAPAR. A person not involved in conduct of the experiment coded (blinded) the treatments solutions with a random letter code. The code was kept secret until all measurements and data processing were finished. Seeds were observed daily for germination and counted only those considered germinated. Seeds were considered germinated when the radicle was at least two millimeters length. Arnica montana 9x increased 5.9\% the seed germination when compared with distilled water.
\end{abstract}

Keywords: Raphanus sativus, high dilutions, germination, homeopathic drugs.

\section{Reference:}

[1] Brazilian Homeopathic Pharmacopoeia, 2nd ed. SP: Atheneu, 1997. Part I. 


\title{
Germinação de sementes de rabanete (Raphanus sativus) tratadas com medicamentos homeopáticos.
}

\begin{abstract}
RESUMO
O ciclo de desenvolvimento do rabanete é considerado curto, uma vez que é colhido em 25-30 dias após semeadura direta. Sob o ponto de vista econômico é uma espécie importante, mas com poucos estudos sobre a germinação de sementes. $\mathrm{O}$ objetivo deste estudo foi avaliar o efeito de substâncias em altas diluições sobre a germinação de sementes de rabanete. O ensaio foi conduzido no Instituto Agronômico do Paraná (IAPAR) em Londrina/Paraná/Brasil. Foi utilizada uma cultivar sem agrotóxicos, chamada Cometa. Os tratamentos foram: Bryonia, solução hidroalcoólica, Arnica montana, Cina e Tremoço+Aveia, todos os tratamentos foram diluídos na 9x; a água destilada e água destilada agitada foram usadas como controles. A tintura mãe de Tremoço+Aveia foi preparada com plantas coletadas na estação experimental do IAPAR em Londrina. A tintura mãe e todos os tratamentos foram preparados de acordo com a Farmacopéia Homeopática Brasileira Parte I [1]. As agitações dos medicamentos foram feitas por um equipamento mecânico, modelo 10-50 Denise fabricado pela Autic. A água foi destilada um dia antes do preparo dos tratamentos. O experimento foi desenvolvido com 300 sementes por tratamento. As sementes foram colocadas em caixa gerbox com papel germitest, sendo utilizados 50 gerbox por tratamento, com seis sementes por gerbox. O papel germitest foi umedecido com os tratamentos e as sementes foram imersas por 2 horas antes da instalação do experimento. As substâncias em altas diluições foram agitadas 100 vezes no equipamento mecânico antes de serem utilizadas para embeber as sementes e umedecer o papel germitest. O experimento foi causalizado e os gerbox foram mantidos sobre a bancada no Laboratório de Proteção de Plantas do IAPAR. Uma pessoa não envolvida na condução do experimento codificou os tratamentos (em cego) com uma letra ao acaso. O código foi mantido em segredo até o processamento final dos dados. As sementes foram observadas diariamente e contadas apenas aquelas consideradas germinadas. As sementes foram consideradas germinadas quando a radícula estava com pelo menos dois milímetros de comprimento. O tratamento Arnica montana 9x aumentou em 5,9\% a germinação quando comparado com a água destilada.
\end{abstract}

Palavras-chave: Raphanus sativus, alta diluição, germinação, medicamento homeopático.

\section{(c)) BY-NC-ND Licensed to GIRI}

Support: authors declare that this study received no funding

Conflict of interest: authors declare there is no conflict of interest

Correspondence author: Solange Monteiro de Toledo Piza Gomes Carneiro, solange_carneiro@iapar.br

How to cite this article: Donadon MFB, Romano EDB, de Pinho WR, de Souza MLV, do Nascimento PHA, Rodrigues MRL, Carneiro SMTPG. Germination of radish seeds (Raphanus sativus) treated with homeopathic drugs. Int J High Dilution Res [online]. 2011 [cited YYYY Month dd]; 10(36): 231-232. Proceedings of the XXV GIRI Symposium and VIII CBFH; 2011 Sep 04-07; Foz do Iguaçu (Brazil). GIRI and ABFH; 2011; Available from: http://www.feg.unesp.br/ ojs/index.php/ijhdr/article/view/471/480 